\title{
Der Schutz geographischer Herkunftsangaben bei Wein in Deutschland von 1894 bis Heute
}

\author{
Hilke Dormann \\ Klaka Rechtsanwälte, Delpstr. 4, 81679 München, Germany
}

\begin{abstract}
Ziel dieses Aufsatzes ist es, einen Überblick über den Schutz geographischer Herkunftsangaben bei Wein in Deutschland zu geben - von den Anfängen 1894 bis zum deutschen Markengesetz von 1995 und der europäischen Verordnung 1308/2013. Es geht darum, die Entwicklung und Ausgestaltung des Schutzes in Deutschland bis Heute darzustellen. Dabei ist auch auf das Verhältnis zwischen europäischem und nationalem Recht zum Schutz geographischer Herkunftsangaben bei Wein einzugehen.
\end{abstract}

\section{Einleitung}

Ende des 19. Jahrhunderts traten mit dem "Gesetz zum Schutze der Waarenbezeichnungen” (WbezG) 1894 [1] und dem "Gesetz zur Bekämpfung des unlauteren Wettbewerbs" (UWG) 1896 [2] die ersten Regelungen in Kraft, die im Deutschen Reich nach heutigem Verständnis einen Schutz geographischer Herkunftsangaben schufen. Von diesem Schutz waren auch geographische Herkunftsangaben bei Wein umfasst. Der nach Meinung der Reichsregierung anhaltende Missbrauch geographischer Herkunftsangaben bei Wein einerseits und die naturgemäß besondere Bedeutung von geographischen Herkunftsangaben bei der Kennzeichnung von Wein anderseits führten jedoch bald nach Einführung des gesetzlichen Schutzes zu Bestrebungen, den Schutz geographischer Herkunftsangaben bei Wein zu verstärken. Die in diesem Zusammenhang eingeführten spezialgesetzlichen Regelungen des Weingesetzes von 1909 (WeinG) [3] wurden diesen Bestrebungen nur begrenzt gerecht. Erst durch diverse Entwicklungen auf internationaler Ebene wurde zu einer entscheidenden Ausdehnung des Schutzes geographischer Herkunftsangaben bei Wein beigetragen. Dies hatte faktisch auch eine Veränderung des gesetzlichen Systems zum Schutz geographischer Herkunftsangaben in Deutschland zur Folge.

Nachfolgend wird in groben Zügen zu erläutern sein, wie der Schutz geographischer Herkunftsangaben in den Gesetzen des gewerblichen Rechtsschutzes von 1894 bis Heute ausgestaltet war (2). Darauf aufbauend ist auf die Besonderheiten, Probleme und Schwachpunkte des Schutzes geographischer Herkunftsangaben im WeinG von 1909 einzugehen. (3). Dies stellt die Grundlage für die darauffolgende chronologische Darstellung der Verstärkung des Schutzes geographischer Herkunftsangaben bei Wein beeinflusst durch internationale Entwicklungen dar - zunächst durch den Versailler Vertrag (VV), den Deutschland 1919 unterzeichnete (4), anschließend durch zwischenstaatliches Recht (5) und die zunehmende Europäisierung des Schutzes geographischer Herkunftsangaben (6).
Die Erkenntnisse und Ergebnisse dieses Aufsatzes stammen aus der Dissertation "Die Geschichte des Schutzes geographischer Herkunftsangaben in Deutschland. Vom Zweiten Deutschen Kaiserreich bis zum Markengesetz 1995" [4]. Dort stellt die Untersuchung des Schutzes geographischer Herkunftsangaben bei Weinen einen bedeutenden Teilaspekt dar.

\section{Der Schutz geographischer Herkunftsangaben in den Gesetzen des gewerblichen Rechtsschutzes von 1894 bis Heute}

\subsection{Geschützte geographische Herkunftsangaben}

Ein lauterkeitsrechtlicher Schutz geographischer Herkunftsangaben, mit dem Zweck, den Verkehr vor Irreführungen zu schützen, wurde in Deutschland erstmals durch das WbezG vom 1. Oktober 1894 eingeführt. $§ 16$ Abs. 1 WbezG gewährte einen strafrechtlichen Schutz, der allerdings auf bestimmte Arten von geographischen Herkunftsangaben beschränkt war, namentlich auf "Staatswappen, Namen oder Wappen eines Ortes, eines Gemeinde- oder weiteren Kommunalverbandes" [5]. Andere als die dort aufgezählten Herkunftsangaben wie beispielsweise Namen von Staaten waren kategorisch vom Schutz nach $\S 16$ Abs. 1 WbezG ausgeschlossen.

Die strafrechtlichen Bestimmungen des $\S 16$ Abs. 1 WbezG wurden durch einen zivilrechtlichen Schutz geographischer Herkunftsangaben nach Inkrafttreten des UWG von 1896 ergänzt. $\S 1$ Abs. 1 S. 1 UWG [6] schützte nach Meinung der zeitgenössischen Literatur jede Art der geographischen Bezeichnung vor einem etwaigen Missbrauch, solange diese als Hinweis auf einen geographischen Ursprung verstanden wurde [7]. Das UWG von 1909 übernahm die Regelung des UWG von 1896 aus $\S 1$ Abs. 1 UWG (1896) in $\S 3$ Abs. 1 UWG [8]. Bis zur Einführung des Markengesetzes 1995 (MarkenG) [9] wurden geographische Herkunftsangaben 
in erster Linie über das UWG von 1909 geschützt. Erst die Markenrechtsreform vom 5. Oktober 1994 führte hundert Jahre nach Inkrafttreten des WbezG dazu, dass der Schutz geographischer Herkunftsangaben im deutschen Recht nicht mehr durch die Vorschriften des UWG, sondern durch die $\S \S 126$ ff. MarkenG gewährt wurde. Die Vorschriften sind im nationalen Recht derzeit die maßgeblichen Regelungen zum Schutz geographischer Herkunftsangaben.

Das MarkenG schützt in $\S 126$ Abs. 1 jede Form eines geographischen Herkunftshinweises, der vom Verkehr als solcher verstanden wird [10] und enthält in $\S 127$ Abs.1 MarkenG einen allgemeinen Irreführungsschutz. Dieser besteht unabhängig davon, ob der Verkehr mit der geographischen Herkunftsangabe weitere Qualitätsmerkmale der gekennzeichneten Ware verbindet [11]. Im Gegensatz dazu gewährt $\S 127$ Abs. 2 MarkenG einen weitergehenden Schutz für solche geographischen Herkunftsangaben, die eine Aussage über die Beschaffenheit der Ware treffen (sog. qualifizierte geographische Herkunftsangaben). Diese dürfen nur dann zur Kennzeichnung von Produkten verwendet werden, wenn diese den mit der Herkunftsangabe verbundenen Produktqualitäten entsprechen [12]. § 127 Abs. 3 MarkenG schützt so genannte geographische Herkunftsangaben mit besonderem Ruf vor einer Verwässerung ihrer Unterscheidungskraft und vor einer etwaigen Rufausbeutung. Dieser Schutz besteht selbst dann, wenn der Verkehr nicht über den wahren geographischen Ursprung der gekennzeichneten Ware in die Irre geführt wird.

\subsection{Abgrenzung von geographischen Herkunfts- und Beschaffenheitsangaben}

Geographische Herkunftsangaben wurden bereits mit Einführung des gesetzlichen Schutzes 1894 abgegrenzt zu bloßen Beschaffenheitsangaben - solchen Herkunftsbezeichnungen also, die nicht als Hinweis auf einen geographischen Ursprung verstanden wurden, sondern lediglich als Hinweis auf eine bestimmte Warenbeschaffenheit. Die Abgrenzung von Herkunfts- und Beschaffenheitsangaben wurde und wird dabei vom Verständnis der angesprochenen Verkehrskreise abhängig gemacht [13]. Beschaffenheitsangaben wurden nicht als geographische Herkunftsangaben geschützt [14]. Auch das gegenwärtig geltende MarkenG schließt in $\S 126$ Abs. 2 Beschaffenheitsangaben vom Schutz geographischer Herkunftsangaben aus.

\section{Geographische Herkunftsangaben im WeinG von 1909}

Folgt man der zeitgenössischen Literatur, war der Missbrauch geographischer Herkunftsangaben zur Kennzeichnung von Wein auch nach Einführung eines gesetzlichen Schutzes desselben in $\S 16$ WbezG und $\S 1$ UWG (1896) weit verbreitet [15]. Dies führte dazu, dass die deutsche Reichsregierung bei der Ausarbeitung des Weingesetzes von 1909 eine Verstärkung des Schutzes geographischer Herkunftsangaben bei Wein beabsichtigte. Weite Teile der Weinwirtschaft befürworteten diese Bestrebungen insbesondere die Weinhändler und -hersteller, die Wein aus bekannten Lagen produzierten bzw. vertrieben und dadurch Umsatzeinbußen erlitten, dass Weine aus weitgehend unbekannten Weinbaugebieten wahrheitswidrig mit den Herkunftsangaben "ihrer" Lagen versehen wurden. So ist zu erklären, dass sich bei den Beratungen zum Weingesetz im deutschen Reichstags gerade die Reichstagsabgeordneten für einen Ausdehnung des Schutzes geographischer Herkunftsangaben bei Wein aussprachen, die für Wahlkreise im Reichstag saßen, welche wichtige Weinbaugebiete in Baden bzw. in der Pfalz darstellten [16]. Gleichzeitig wurde eine etwaige Ausdehnung des Schutzes geographischer Herkunftsangaben bei Weinen von einer nicht unerheblichen Zahl von Weinhändlern und -herstellern kritisiert - namentlich von denjenigen, die Weine aus weitgehend unbekannten Weinbaugebieten herstellten bzw. vertrieben und von dem guten Ruf der Weinbaugebiete durch den wahrheitswidrigen Gebrauch geographischer Bezeichnung zu profitieren versuchten.

Die schließlich eingeführten Bestimmungen eines spezialgesetzlichen Schutzes geographischer Herkunftsangaben für Wein in $\S 6$ des Weingesetzes von 1909 stellten letztendlich einen Kompromiss zwischen diesen verschiedenen Interessen dar. Grundsätzlich begründete $\S 6$ Abs. 1, Abs. 2 S. 1 WeinG eine "allgemeine Wahrheitspflicht" bei der Verwendung geographischer Herkunftsangaben zur Kennzeichnung von Wein. Unabhängig vom Verständnis des Verkehrs mussten diese den wahren geographischen Ursprung eines Weins kennzeichnen. Eine Verwendung von geographischen Bezeichnungen als Beschaffenheitsangabe, wie es die Bestimmungen des WbezG und des UWG in Deutschland durchaus ermöglichten, war ausgeschlossen:

(1) "Im gewerbsmäßigen Verkehre mit Wein dürfen geographische Bezeichnungen nur zu Kennzeichnung der Herkunft verwendet werden.

(2) Die Vorschriften des $\S 16$ Abs. 2 des Gesetzes zum Schutz der Warenbezeichnungen vom 12. Mai 1894 (Reichs-Gesetzbl. S. 145) und des $\S 1$ Abs. 3 des Gesetzes zur Bekämpfung des unlauteren Wettbewerbes vom 27. Mai 1896 (Reichs-Gesetzbl. S. 145) finden auf die Benennung von Wein keine Anwendung" [17].

Von diesem Grundsatz der Wahrheitspflicht machte allerdings $\S 6$ Abs. 2 S. 2 WeinG eine entscheidende Ausnahme:

"Gestattet bleibt jedoch, die Namen einzelner Gemarkungen oder Weinbergslagen, die mehr als einer Gemarkung angehören, zu benutzen, um gleichartige und gleichwertige Erzeugnisse benachbarter oder nahegelegener Gemarkungen oder Lagen zu bezeichnen" [18].

Die Rechtsprechung legte diese Ausnahmeregelung nach Inkrafttreten des Weingesetzes 1909 weit aus. Dies führte unter anderem dazu, dass nicht nur wie es der Wortlaut von $\S 6$ Abs. 2 S. 2 WeinG vermuten ließ - Namen einzelner Weingemarkungen [19] sowie einzelner Weinbergslagen [20], die mehr als einer Gemarkung angehörten, wahrheitswidrig verwendet werden durften. Vielmehr hielt die Rechtsprechung eine solche Kennzeichnung von Wein mit Namen von 
Weinbergslagen auch dann für zulässig, wenn diese gerade nicht mehr als einer Gemarkung angehörten, dafür aber in Verbindung mit dem Namen der Gemarkung verwendet wurden, zu der die betreffende Weinbergslage gehörte [21]. Dies führte letzten Endes dazu, dass die eigentlich in $\S 6$ Abs. 1 WeinG aufgestellten Wahrheitspflichbei der Verwendung geographischer Bezeichnungen deutlich eingeschränkt wurde und von dem ursprünglichen Vorhaben der Reichsregierung, den Schutz geographischer Herkunftsangaben bei Wein durch spezialgesetzliche Regelungen deutlich zu verstärken, nicht viel übrig blieb.

\section{Der Schutz geographischer Herkunftsangaben bei Weinen im Versailler Vertrag und dessen Auswirkung auf das deutsche Recht}

\subsection{Inhalt des Art. $275 \mathrm{VV}$}

Nach Unterzeichnung des Versailler Vertrags am 28. Juni 1919 war neben der einseitigen Verpflichtung in Art. $274 \mathrm{VV}$, geeignete Maßnahmen zum Schutz vor unlauterem Wettbewerb und vor einem Missbrauch geographischer Herkunftsangaben zu treffen, insbesondere Art. 275 VV Gegenstand juristischer Kontroversen in Deutschland. Die Vorschrift richtete sich gegen den spezifischen Missbrauch geographischer Herkunftsangaben bei Weinen und "geistigen Getränken". Sie zwang Deutschland unter der Bedingung einer entsprechenden Gegenseitigkeitserklärung, die Rechtslage eines alliierten oder assoziierten Landes diesbezüglich anzuerkennen und erklärte in S. 1:

"Deutschland verpflichtet sich unter der Bedingung der Gegenseitigkeit auf diesem Gebiet, die in einem alliierten oder assoziierten Lande geltenden und durch die zuständigen Behörden Deutschland gehörig bekanntgegebenen Gesetze und in Übereinstimmung mit diesen Gesetzen ergangenen Verwaltungs- oder Gerichtsentscheidungen $\mathrm{zu}$ beobachten, wodurch das Recht auf eine Lagebezeichnung für die in dem betreffenden Lande erzeugten Weine oder geistigen Getränke bestimmt oder geregelt wird oder wodurch die Bedingungen bestimmt oder geregelt werden, an welche die Erlaubnis zum Gebrauch einer Lagebezeichnung geknüpft ist" [22].

Am 2. Juni 1920 gab Frankreich eine entsprechende Gegenseitigkeitserklärung ab [23]. Portugal folgte diesem Beispiel mit einer Erklärung am 3. September 1921 [24]. Dies führte dazu, dass der Schutzumfang geographischer Herkunftsangaben bei Weinen gewissermaßen von der "Nationalität" einer Herkunftsangabe abhing. Ob ein Missbrauch geographischer Herkunftsangaben vorlag, richtete sich in Deutschland bei französischen Herkunftsangaben nach französischem Recht, bei portugiesischen Herkunftsangaben nach portugiesischem Recht und bei allen übrigen Herkunftsangaben nach deutschem Recht.

Frankreich selbst stellte den Gebrauch geographischer Bezeichnungen bei Weinen unter die ausnahmslose Bedingung der Wahrheitspflicht. Sie durften nur verwendet werden, um auf den wahren geographischen Ursprung des Weins hinzuweisen [25]. Der Versailler Vertrag i.V.m. den entsprechenden Gegenseitigkeitserklärungen hatte also für
Deutschland zur Folge, dass bei der Kennzeichnung von Weinen mit französischen Bezeichnungen eine absolute Wahrheitspflicht eingeführt wurde. Im portugiesischen Recht war die Situation ähnlich wie in Frankreich allerdings beschränkt auf die Bezeichnung "Madeira-" und "Portwein". Diese durften ausschließlich als Hinweis auf den geographischen Ursprung des likörhaltigen Weins, also ebenfalls nicht wahrheitswidrig verwendet werden [26]. Das hatte Deutschland zukünftig beim Schutz geographischer Herkunftsangaben zu berücksichtigen.

\subsection{Auswirkungen auf das deutsche Recht}

\subsubsection{Die Reform des Weing vom 1. Februar 1923}

Durch Art. 275 VV i.V.m. mit den Gegenseitigkeitserklärungen Portugals und Frankreich wurden strengere Maßstäbe an die Zulässigkeit geographischer Herkunftsangaben bei der Kennzeichnung von Wein als im bisherigen deutschen Weinrecht gestellt. Die Ausnahmen vom Grundsatz der Wahrheitspflicht bei der Verwendung geographischer Bezeichnungen für "Namen einzelner Gemarkungen oder Weinbergslagen, die mehr als einer Gemarkung angehören" (§ 6 Abs. 2 S. 2 WeinG), waren mit dem französischen und portugiesischem Recht nicht vereinbar.

Der deutsche Gesetzgeber führte daher in der Reform des WeinG vom 1. Februar 1923 § 7a WeinG ein. Die Vorschrift enthielt eine zusätzliche Regelung über die Verwendung portugiesischer und französischer Bezeichnungen zur Kennzeichnung von Wein und besagte, dass $\oint 6$ Abs. 2 S. 2 WeinG "nicht für die Verwendung von französischen und portugiesischen geographischen Bezeichnungen" gelte [27]. Damit durften französische und portugiesische Herkunftsangaben auch nach dem deutschen WeinG nicht wahrheitswidrig zur Kennzeichnung von Weinen verwendet werden. Im Übrigen blieben die Bestimmungen des $\S 6$ WeinG bestehen. Weine konnten also weiterhin mit anderen als französischen und portugiesischen geographischen Bezeichnungen unter den Voraussetzungen des $\S 6$ Abs. 2, S. 2 WeinG versehen werden, um auf einen anderen als den wahren Ursprung des Weins hinzuweisen. Portugiesische und französische Bezeichnungen bei Weinen unterlagen einem stärkeren gesetzlichen Schutz als geographische Bezeichnungen anderer "Nationalität".

Nach der Unterzeichnung des Versailler Vertrags und den entsprechenden Gegenseitigkeitserklärungen Frankreichs und Portugals hatte es in der zeitgenössischen Literatur durchaus Bestrebungen gegeben, die Verpflichtung zum ausnahmslosen Schutz französischer und portugiesischer Bezeichnungen bei Weinen als Chance für eine grundlegende Schutzausdehnung zu begreifen und allgemein eine Wahrheitspflicht bei der Verwendung geographischer Bezeichnungen zur Kennzeichnung von Wein einzuführen - unabhängig davon, ob es sich im konkreten Fall um französische oder portugiesische Herkunftsangaben oder aber um geographische Herkunftsangaben anderer "Nationalität" handelte [28]. Diese Überlegungen konnten sich gegenüber dem Gesetzgeber jedoch nicht durchsetzen. Die Bestimmungen des Weingesetzes wurden nur so weit geändert, wie es Art. 275 
VV i.V.m. der Gegenseitigkeitserklärung Frankreichs und Portugals verlangte.

\subsubsection{Der Beitritt zum Madrider Herkunftsabkommen am 21. März 1925}

Als Folge des Versailler Vertrags unterzeichnete Deutschland 1925 das Madrider Herkunftsabkommen (MHA), das zu einer weiteren Verstärkung des Schutzes geographischer Herkunftsangaben bei Wein führte. Das Abkommen verpflichtete dazu, Waren bei einem Missbrauch geographischer Herkunftsangaben unmittelbarer oder mittelbarer Art zu beschlagnahmen. Deutschland hatte sich vor dem Ersten Weltkrieg wiederholt geweigert, diesem Abkommen beizutreten. Das war in erster Linie auf die Regelung des Art. 4 MHA zurückzuführen, der folgende Bestimmungen enthielt:

"Die Gerichte jedes Landes haben zu entscheiden, welche Bezeichnungen infolge ihres gemeinen Charakters nicht unter die Bestimmungen des gegenwärtigen Abkommens fallen. Die Lagebezeichnungen der Erzeugnisse des Weinbaues werden jedoch nicht von der in diesem Artikel aufgestellten Ausnahme umfasst" [29].

Zwar hing gem. Art. 4 S. 1 MHA die Frage, ob eine schutzfähige geographische Herkunftsangabe oder eine lediglich beschreibende geographische Beschaffenheitsangaben vorlag, von der rechtlichen Beurteilung des Mitgliedsstaates $a b$, in dem der Missbrauch geographischer Herkunftsangaben im konkreten Fall gerichtlich überprüft wurde. Art. 4 S. 2 MHA machte hiervon jedoch eine entscheidende Ausnahme und schloss eine Umwandlung von Lagenamen bei Weinerzeugnissen in nicht schutzfähige Beschaffenheitsangaben kategorisch aus. Dies war mit den deutschen Regelungen zum Schutz geographischer Herkunftsangaben bei Wein im Weingesetz von 1909 nicht vereinbar. § 6 Abs. 2 S. 2 WeinG sah gerade die Möglichkeit vor, Lagenamen wahrheitswidrig bei der Kennzeichnung von Wein zu verwenden.

Ausschlaggebend für die Weigerung Deutschlands, dem Madrider Herkunftsabkommen beizutreten, waren aber nicht die Bestimmungen des deutschen Weingesetzes gewesen, sondern die Tatsache, dass man bis zur Unterzeichnung des Versailler Vertrags im Deutschen Reich die Auffassung vertrat, die Bezeichnung "Cognac" sei eine bloße Beschaffenheitsangabe für einen vollständig aus Wein hergestellten Weinbrand und enthalte keinen Hinweis auf die région de Cognac [30]. Dementsprechend wurde diese Bezeichnung von deutschen Weinbrandherstellern und -händlern zur Kennzeichnung von Weinbrand verwendet - unabhängig von dessen Herkunft. Dies war nicht mit den Vorgaben von Art. 4 S. 2 MHA vereinbar, der nicht nur auf Wein, sondern auf sämtliche Weinbauerzeugnisse Anwendung fand. Für das Deutsche Reich war dies ausschlaggebend gewesen, sich dem MHA nicht anzuschließen [31].

Der Versailler Vertrag und die nachfolgende Gegenseitigkeitserklärung Frankreichs hatten jedoch auch dazu geführt, dass die Bezeichnung "Cognac" für deutschen Weinbrand nicht länger zulässig war und ausschließlich für vollständig in der région de Cognac hergestellte Weinbrände verwendet werden durfte [32]. Dementsprech end war der für Deutschland bis dato entscheidende Grund weggefallen, dem Madrider Herkunftsabkommen nicht beizutreten. Deutschland unterzeichnete das Abkommen am 21. März 1925 [33].

Damit durften Lagebezeichnungen bei Wein nicht mehr länger wahrheitswidrig verwendet werden. Auch insofern führte der Versailler Vertrag - als Hauptursache für den Beitritt Deutschlands zum Madrider Herkunftsabkommen - zu einer Verstärkung des Schutzes geographischer Herkunftsangaben bei Wein. Freilich blieb dem deutschen Recht die Auslegung des Begriffs "Lagebezeichnung" in Art. 4 S. 2 MHA und damit auch die Bestimmung, welche Bezeichnung bei Weinen von der allgemeinen Wahrheitspflicht umfasst werden, überlassen.

\section{Der Schutz geographischer Herkunftsangaben auf internationaler Ebene}

Nach Ende des Zweiten Weltkriegs versuchte die Bundesrepublik Deutschland vermehrt, den Schutz geographischer Herkunftsangaben auf internationaler Ebene $\mathrm{zu}$ verstärken. Hiervon profitierte auch der Schutz geographischer Herkunftsangaben bei Wein.

Hauptproblem bei der Frage, wie eine solche Schutzausdehnung umzusetzen sei, war dabei, dass geographische Herkunftsangaben in den verschiedenen Staaten durch zwei sich grundlegend unterscheidende Systeme geschützt wurden. Während die Bundesrepublik geographische Herkunftsangaben in erster Linie wettbewerbsrechtlich schützte, hatten andere Staaten hierzu gehörten Frankreich, Italien, Spanien und Portugal - sich für einen zweigleisigen Schutz geographischer Herkunftsangaben entschieden. Neben dem lauterkeitsrechtlichen Schutz für herkömmliche Herkunftsangaben existierte in diesen Staaten ein zusätzlicher Schutz für Ursprungsbezeichnungen, die neben einem Hinweis auf den geographischen Ursprung auch einen Hinweis auf besondere, geographisch bedingte Eigenschaften enthielten [34]. Diese Ursprungsbezeichnungen waren in der Regel in irgendeiner Form ausdrücklich als solche in den Staaten anerkannt - sei es durch gesetzlichen, gerichtlichen oder behördlichen Akt. So wurde in Frankreich bereits 1935 das Institut National des Appellations d'Origine des vins et des eaux-de-vie (INAO) als zentrale Behörde gegründet, bei der für Wein und Lebensmittel der Schutz als Ursprungsbezeichnung beantragt und registriert wurde. Durch diese ausdrückliche Anerkennung gewährten Ursprungsbezeichnungen dem rechtmäßigen Verwender ein subjektives Ausschließlichkeitsrecht und stellten daher eine Form geistigen Eigentums dar [35].

Auf multilateraler Ebene wurde mehrfach vergeblich versucht, diese beiden unterschiedlichen Schutzsysteme in Einklang miteinander zu bringen. Da man dennoch den Schutz geographischer Herkunftsangaben verstärken wollte, schlugen die Staaten - in Abhängigkeit von der nationalen Ausgestaltung des Schutzsystems unterschiedliche Wege ein, um eine Schutzausdehnung zu erreichen.

Diejenigen Staaten, die auf nationaler Ebene einen zweigleisigen Schutz geographischer Herkunftsangaben 
verfolgten, schlossen sich 1958 zum so genannten Lissaboner Ursprungsabkommen (LUA) zusammen [36]. Frankreich, Israel, Italien, Kuba, Portugal, Rumänien, Spanien, die Tschechoslowakei und Ungarn unterzeichneten am 31. Oktober 1958 das Abkommen und trugen so zu einer Verstärkung des Schutzes von Ursprungsbezeichnungen bei. Das Abkommen verpflichtete dazu, diejenigen Ursprungsbezeichnungen der anderen Mitgliedstaaten zu schützen, die im Ursprungsland durch gesetzlichen, gerichtlichen oder behördlichen Akt anerkannt waren.

Für die Bundesrepublik Deutschland, dessen nationales Recht keinen Schutz von Ursprungsbezeichnungen kannte, machte die Unterzeichnung des LUA keinen Sinn. Dennoch war man bestrebt, einen international stärkeren Schutz geographischer Herkunftsangaben zu erreichen, der die Eigenheiten des deutschen Rechts beim Schutz geographischer Herkunftsangaben aber nicht vernachlässigte. Deutschland unterzeichnete daher mit Beginn der 1960er Jahre bis hinein in die 1980er Jahre eine Reihe von bilateralen Verträgen. Den ersten eines solchen Vertrags schloss Deutschland am 8. März 1960 mit Frankreich ab [37]. Es folgten Verträge mit Italien [38] Griechenland [39], der Schweiz [40], Spanien [41] und schließlich mit Österreich am 6. Oktober 1981 [42]. Letzterer ist bis heute noch nicht ratifiziert [43]. Im Übrigen sind diese Verträge aber aktuell in Kraft.

Kennzeichnend für diese Verträge war erstens, dass sowohl gewöhnliche Herkunftsangaben als auch Ursprungsbezeichnungen geschützt wurden. Zweitens durften die von den Verträgen geschützten Angaben nur zur Kennzeichnung von Waren und Erzeugnissen des Ursprungslands verwendet werden. Drittens wurden die von den Verträgen geschützten Angaben in Listen erfasst, die den Verträgen als Anlagen beigefügt wurden. Die in den Listen aufgeführten Bezeichnungen wurden nach Art der gekennzeichneten Waren untergliedert. Einen wesentlichen Bestandteil dieser Listen nahmen dabei die unter der Kategorie "Wein" aufgeführten Erzeugnisse ein. Viertens richtete sich der Umfang des Schutzes einer durch die Verträge geschützten Angabe nach dem Recht des Ursprungslands und nicht nach dem Recht des Schutzlands (sog. Ursprungslandprinzip). So umging man die Problematik, dass der Schutz geographischer Herkunftsangaben in den verschiedenen Staaten unterschiedlich ausgestaltet war. Fünftens wurde der Schutz der listenmäßig erfassten geographischen Herkunftsangaben durch eine Generalklausel ergänzt, die den Gebrauch von Kennzeichen, Marken, Namen, Aufschriften oder Abbildungen verbot, die unmittelbar oder mittelbar falsche oder irreführende Angaben über Herkunft, Ursprung, Natur, Sorte oder wesentliche Eigenschaften der Erzeugnisse oder Waren enthielten. Sechstens bestimmten die bilateralen Verträge "neueren Typs", dass geographische Bezeichnungen auch dann geschützt waren, wenn diese "in Übersetzung oder mit Hinweis auf die tatsächliche Herkunft oder mit Zusätzen wie "Art", "Typ", "Fasson", "Nachahmung" oder dergleichen benutzt werden" [44]. Die galt unabhängig davon, ob im konkreten Fall eine Ursprungsbezeichnung oder eine gewöhnliche Herkunftsangabe verwendet worden war [45].
Die Unterzeichnung der Verträge führte auf internationaler Ebene nicht nur zu einer Verstärkung des Schutzes geographischer Herkunftsangaben im Allgemeinen, sondern - da ebenfalls Gegenstand der vertraglichen Regelungen - dehnte auch den Schutz geographischer Herkunftsangaben bei Weinen maßgeblich aus. Die Verträge stellten eine Möglichkeit dar, einen effektiven Schutz geographischer Herkunftsangaben in anderen Ländern zu erreichen und brachten gleichzeitig die unterschiedlichen Schutzsysteme in den verschiedenen Ländern miteinander in Einklang. Ferner führten die Verträge erstmals dazu, dass bei Weinen aus Deutschland bestimmte Arten von geographischen Herkunftsangaben listenmäßig festgehalten wurden. Eine solche Auflistung - wie sie beispielsweise in Frankreich durch die Verwaltungsbehörde INAO (s.o.) seit 1935 stetig erfolgte - war dem wettbewerbsrechtlich ausgestalteten Schutz geographischer Herkunftsangaben in Deutschland bisher fremd.

\section{Europäisierung des Schutzes geographischer Herkunftsangaben}

Seit der zweiten Hälfte des 20. Jahrhunderts wurde die Ausgestaltung des Schutzes geographischer Herkunftsangaben in Deutschland mehr und mehr durch europäische Verordnungen beeinflusst. Bereits ab den 1960er Jahren traten Verordnungen in Kraft, die Vorschriften über geographische Herkunftsangaben bei Wein enthielten [46]. Diese Regelungen waren jedoch nur von untergeordneter Bedeutung und bezogen sich ausschließlich auf die Kennzeichnung von Verschnittweinen (vgl. z.B. Art. 30 Abs. 3 der VO (EWG) 816/70). Einen Schritt weiter ging dagegen die VO (EWG) Nr. 2081/92, die einen umfassenden Schutz geographischer Herkunftsangaben auf dem europäischem Markt bezweckte [47]. Gegenstand dieser Verordnung waren jedoch Agrarerzeugnisse und Lebensmittel im Allgemeinen. Weine wurden von der Verordnung explizit ausgenommen. Allerdings traf die 2008 verabschiedete VO (EG) 479/2008 [48] (sowie die hierauf aufbauenden nachfolgenden europäischen Verordnungen) weitgehend inhaltsgleiche Regelungen zum Schutz geographischer Herkunftsangaben bei Wein wie die VO (EWG) 2081/1992. Es ist daher nachfolgend auf die Bestimmungen der VO (EWG) Nr. 2081/92 und die Besonderheiten des hierauf basierenden produktspezifischen europäischen Schutzes für Wein einzugehen, um anschließend darzustellen, in welchem Verhältnis die europäischen Bestimmungen zum deutschen Schutz geographischer Herkunftsangaben stehen.

\subsection{Die VO (EWG) Nr. 2081/92 zum Schutz von geographischen Herkunftsangaben und Ursprungsbezeichnungen für Agrarerzeugnisse und Lebensmittel}

Voraussetzung für den Schutz durch die 1992 verabschiedete Verordnung war ein mehrstufiges Registrierungsverfahren, von dem ein Teil der Verfahrensschritte bei den nationalen Fachbehörden - in Deutschland also beim Patent- und Markenamt - erfolgen konnte. Mit Inkrafttreten des MarkenG 1995 schuf der Gesetzgeber durch 
die $\S \S 130$ bis 136 MarkenG die notwendigen nationalen Vorschriften, die Verfahren, Überwachung und Sanktionen für die Registrierungsschritte beim Patent- und Markenamt festlegten. Die VO (EWG) 2081/92 wurde zunächst durch die VO (EG) 510/06 [49] und später durch die VO (EU) 115/2012 [50] ersetzt.

Die VO Nr. 2081/92 differenzierte zwischen Ursprungsbezeichnungen und geographische Angaben. Sowohl bei Ursprungsbezeichnungen als auch bei geographischen Angaben wurde der Schutz an zwei wesentliche Voraussetzungen geknüpft. Zum einen setzte Art. 2 VO voraus, dass es sich um den Namen "einer Gegend, eines bestimmten Ortes oder in Ausnahmefällen eines Landes [handelt], der zur Bezeichnung eines Agrarerzeugnisses oder eines Lebensmittels dient, das aus dieser Gegend, diesem bestimmten Ort oder diesem Land stammt". Zum anderen knüpfte Art. 2 VO den Schutz an die zusätzliche Voraussetzung, dass die geographische Angabe oder Ursprungsbezeichnung mit einer bestimmten Warenqualität verbunden war. Welche Anforderungen an derartige Qualitätsfaktoren zu stellen waren, machte Art. 2 VO davon abhängig, ob es um den Schutz einer geographischen Angabe oder einer Ursprungsbezeichnung ging. Letztere musste gemäß Art. 2 Abs. 2 Buchst. a VO der Bezeichnung von Erzeugnissen dienen, die ihre "Güte oder Eigenschaften überwiegend oder ausschließlich den geographischen Verhältnissen einschließlich der natürlichen und menschlichen Einflüsse" verdankte und die "in dem begrenzten geographischen Gebiet erzeugt, verarbeitet und hergestellt" wurde. Im Gegensatz dazu erforderte der Schutz einer geographischen Angabe lediglich den Nachweis, dass sich für die gekennzeichneten Erzeugnisse "eine bestimmte Qualität, das Ansehen oder eine andere Eigenschaft" aus dem angegebenen "geographischen Ursprung ergibt und dass das Ursprungserzeugnis in dem begrenzten geographischen Gebiet erzeugt und/oder verarbeitet und/oder hergestellt wurde" (Art. 2 Abs. 2 Buchst. b VO). Ursprungsbezeichnungen und geographische Angaben wurden durch Art. 13 VO vor jedwedem Missbrauch geschützt und durften nur zur Kennzeichnung von Waren verwendet werden, wenn diese dem angegebenen Ursprung und den im Registrierungsverfahren anzugebenen Qualitätskriterien entsprachen.

Damit wurde durch die VO (EWG) Nr. 2081/92 auf europäischer Ebene ein Schutz von geographischen Bezeichnungen (als Oberbegriff für Ursprungsbezeichnungen und geographische Angaben) eingeführt, wie ihn auch die Staaten mit einem zweigleisigen Schutz beim Schutz von Ursprungsbezeichnungen kannten. Die Verordnung knüpfte ihren Schutz nicht nur an objektiv zu beurteilende Qualitätskriterien, sondern auch an ein durchzuführendes Registrierungssystem, wie es beispielsweise auch in Frankreich bestand (s.o.).

\subsection{Produktspezifischer Schutz geographischer Herkunftsangaben bei Wein in der Europäischen Union}

Am Muster der VO (EWG) 2081/92 orientiertierend, verabschiedete die Europäische Gemeinschaft 2008 die VO
(EG) 479/2008, die ebenfalls mittels Registrierungsverfahren einen europäischen Schutz für geographische Angaben und Ursprungsbezeichnungen in den Art. 34 ff. gewährte. Wie bereits die Verordnung für Lebensmittel von 1992 gewährte auch diese Verordnung nur dann einen Schutz, wenn die geographische Bezeichnung mit bestimmten objektiven Qualitätskriterien verbunden war. Hinsichtlich der Anforderungen an diese Kriterien unterschieden sich die Weinverordnung und die Verordnung für Lebensmittel allerdings (siehe sogleich). Die VO (EG) 479/2008 wurde durch die VO (EG) 1234/2007 in der Fassung der VO (EG) 491/2009 [51] und später durch die VO (EU) 1308/2013 [52] ersetzt. Letztere ist das derzeit maßgebliche Regelungswerk für den Schutz geographischer Herkunftsangaben bei Wein. Wenngleich sich dies nicht aus den Vorschriften des MarkenG ergibt, fanden und finden die $\S \S 130$ ff. MarkenG über das Registrierungsverfahren von Herkunftsangaben auch für die verschiedenen Weinverordnungen Anwendung. Die Bestimmungen zum Schutz geographischer Herkunftsangaben bei Wein in diesen Verordnungen sind weitestgehend inhaltsgleich. Der Einfachheit halber wird daher lediglich anhand der aktuell geltenden VO (EU) 1308/2013 dargestellt, wie dieser europäische Schutz ausgestaltet ist.

Auch die VO (EU) 1308/2013 setzt für den Schutz von Ursprungsbezeichnungen und geographischen Angaben gleichermaßen voraus, dass es sich um den Namen "einer Gegend, eines bestimmten Ortes oder in ordnungsgemäß gerechtfertigten Ausnahmefällen eines Landes [handelt], der zur Bezeichnung eines [... Weinbauerzeugnisses] dient" (Art. 93). Hinsichtlich der Anforderungen, die an die Qualitätsfaktoren des Weinbauerzeugnisses bei Ursprungsbezeichnungen zu stellen sind, verlangt Art. 93 Abs. 1 lit. a:

i) "Es verdankt seine Güte oder Eigenschaften überwieg end oder ausschließlich den geographischen Verhältnis sen einschließlich der natürlichen und menschlichen Einflüsse;

ii) die Weintrauben, aus denen es gewonnen wird, stammen ausschließlich aus diesem geographischen Gebiet;

iii) seine Herstellung erfolgt in diesem geographischen Gebiet und

iv) es wurde aus Rebsorten gewonnen, die zu Vitis vinifera gehören".

Bei geographischen Angaben müssen Weinbauerzeugnisse gem. Art. 93 Abs. 1 lit. b folgende Qualitätsfaktoren einhalten:

i) "Es verdankt seine Güte, ein bestimmtes Ansehen oder andere Eigenschaften, die sich aus diesem geographischen Ursprung ergeben;

ii) mindestens $85 \%$ der $\mathrm{zu}$ seiner Handlung verwendeten Trauben stammen ausschließlich aus diesem geographischen Gebiet;

iii) seine Herstellung erfolgt in diesem geographischen Gebiet und

iv) es wurde aus Rebsorten gewonnen, die zu Vitis vinifera oder einer Kreuzung zwischen der Sorte Vitis vinifera und einer anderen Sorte der Gattung Vitis gehören". 
Wie in der Verordnung von 1992 für Lebensmittel werden Ursprungsbezeichnungen und geographische Angaben gleichermaßen vor jedem Missbrauch geschützt und dürfen ausschließlich zur Kennzeichnung von Waren verwendet werden, wenn diese dem angegebenen Ursprung und den im Registrierungsverfahren anzugebenen Qualitätskriterien entsprechen (Art. 103).

\subsection{Verhältnis des europäischen Rechts zum deutschen Schutz geographischer Herkunftsangaben}

Seit 1995 werden geographische Herkunftsangaben auf nationaler Ebene über die $\S \S 126$ ff. MarkenG geschützt (s.o.). Dieser Schutz weicht in vielerlei Hinsicht von dem Schutz durch die europäische VO (EU) 1308/2013 ab. Ein Registrierungsverfahren von geographischen Herkunftsangaben wie es auf europäischer Ebene besteht, ist dem deutschen Recht fremd. Daneben ist der Schutz geographischer Herkunftsangaben in $\S 127$ Abs. 1 MarkenG wettbewerbsrechtlich ausgestaltet und knüpft nicht an detaillierte Qualitätsvorgaben an wie die Verordnung in Art. 93. Allerdings macht $\S 127$ Abs. 2 MarkenG wenngleich nicht in derart detaillierter Form, so doch ähnlich wie das europäische Recht - den Schutz der sog. qualifizierten geographischen Herkunftsangaben davon abhängig, dass die Herkunftsangabe einen objektiven Hinweis auf die Qualität der gekennzeichneten Waren enthält. Insofern sind Ähnlichkeiten zum europäischen Recht vorhanden.

Es stellt sich daher die Frage, in welchem Verhältnis der Schutz geographischer Herkunftsangaben durch das europäische Recht zu dem Schutz des deutschen MarkenG steht. Da die VO (EWG) 2081/1992 für Lebensmittel und Agrarerzeugnisse bereits sechzehn Jahre vor der ersten europäischen Verordnung über den Schutz geographischer Angaben und Ursprungsbezeichnungen bei Wein (VO (EG) 479/2008) in Kraft trat, wurde diese Frage in der Rechtsprechung auch anhand der Verordnung von 1992 geklärt. Da sich diese in ihrer Systematik aber - wie dargestellt - weitestgehend mit den Weinverordnungen zum Schutz geographischer Angaben und Ursprungsbezeichnungen deckt, sind die von der Rechtsprechung aufgestellten Grundsätze auf das Verhältnis der derzeit geltenden VO (EU) 1308/2013 für Wein zum deutschen MarkenG zu übertragen [53].

Für herkömmliche geographische Herkunftsangaben, die nicht mit objektiven Qualitätskriterien der Ware verbunden sind und für die der Anwendungsbereich des europäischen Rechts daher nicht eröffnet ist, wurde bereits kurz nach Inkrafttreten der VO (EWG) 2081/1992 festgestellt, dass diese auf nationaler Ebene weiterhin geschützt würden [54]. Ebenso bestand Einigkeit, dass eine nach europäischem Recht eingetragene Herkunftsangabe sich nicht auf den Schutz durch nationale oder zwischenstaatliche Vorschriften berufen könne [55].

Lange Zeit war dagegen umstritten, ob sich solche geographischen Herkunftsangaben, die zwar theoretisch die Voraussetzungen für einen Schutz durch das europäische Recht erfüllten, die mangels Registrierung tatsächlich aber nicht von dessen Schutz umfasst waren, auf einen Schutz durch das deutsche Recht oder auch durch bilaterale
Staatsverträge berufen können [56]. Der EuGH stellte insoweit einen abschließenden Charakter des europäischen Rechts fest [57]. Das bedeutet, dass solche geographischen Herkunftsangaben keinen Schutz durch nationales deutsches oder zwischenstaatliches Recht beanspruchen können. Dies gilt auch für Herkunftsangaben, die zwar bereits für eine Registrierung angemeldet, aber noch nicht eingetragen sind [58].

Hieraus folgt, dass für den Schutz geographischer Herkunftsangaben bei Weinen in erster Linie das europäische Recht maßgeblich ist und das deutsche Recht entscheidend an Bedeutung verloren hat. Lediglich herkömmliche Herkunftsangaben werden weiterhin durch das nationale Recht geschützt. Gleichzeitig hat dieser Anwendungsvorrang des europäischen Rechts dazu geführt, dass das dort bestehende Registrierungssystem geographischer Herkunftsangaben gewissermaßen auch in Deutschland Einzug erhalten hat.

\section{Literaturhinweise}

[1] RGB1. (1894), S. 441

[2] RGBl. (1896), S. 145

[3] RGBl. (1909), S. 499

[4] H. Kickler, Die Geschichte des Schutzes geographischer Herkunftsangaben in Deutschland, Tübingen (2012)

[5] RGBl. (1894), S. 441

[6] RGBl. (1896), S. 145

[7] J. Bachem/H. Roeren, Das Gesetz zur Bekämpfung des unlauteren Wettbewerbs (1896), S. 57; C. Finger, Reichsgesetz zur Bekämpfung des unlauteren Wettbewerbs vom 27. Mai 1896, Berlin (1907), S. 46; D. Grünewald, Das Gesetz zur Bekämpfung des unlauteren Wettbewerbs vom 27. Mai 1896, 2. Aufl., München (1896), S. 12; A. Lobe, Das Gesetz. zur Bekämpfung des unlauteren Wettbewerbes vom 27. Mai 1896, Leipzig (1896), S. 63; A. Pinner, Das Reichsgesetz zur Bekämpfung des unlauteren Wettbewerbs vom 27. Mai 1896, Berlin (1903), S. 37; P. Schmid, Die Gesetze zum Schutz des gewerblichen Eigentums, Berlin (1897), S. 298; M. Wassermann, Der Schutz der Herkunftsbezeichnung in Deutschland, in: MuW 8 (1907/1908), S. 187-193 (187)

[8] RGBl. (1909), S. 499

[9] BGBl. I (1994), S. 3082; BGBl. I (1995), S. 156

[10] R. Ingerl/C. Rohnke, Markengesetz, 3. Aufl. (2010), $\S 126$, Rn. 4-7; K.H. Fezer, Markengesetz, 4. Aufl. (2009), § 126, Rn. $10 \mathrm{f}$

[11] BGH, in: GRUR (2002), S. 160-163 (161)

[12] K.-H. Fezer, Markengesetz, 3. Aufl. (2001), § 126, Rn. 7

[13] Zum alten Recht: P. Allfeld, Kommentar zu den Gesetzen über das gewerbliche Urheberrecht, München (1904), S. 640 f.; G. Freund/J. Magnus, Gesetz zum Schutz der Warenbezeichnungen vom 12. Mai 1894, Berlin (1909), S. 218; A. Seligsohn, Gesetz zum Schutz der Warenbezeichnungen, 2. Auflage, Berlin (1905), S. 223; RG, in: Blatt für Patent-, Muster- und Zeichenschutz 6 (1900), S. 328331; 8 (1902), S. 32; in: Juristische Wochenzeitschrift 
(1901), S. 851. Zum gegenwärtigen Recht: F. Ekey/D. Klippel/A. Bender, Heidelberger Kommentar zum Markenrecht, 2. Aufl., Heidelberg 1 (2009), $\S$ 126, Rn. 6; R. Knaak, Der Schutz geographischer Herkunftsangaben im neuen Markengesetz, in: GRUR (1995), S. 103-112 (105); H. Helm, Der Schutz geographischer Herkunftsangaben nach dem Markengesetz, in: Festschrift für Vieregge, Berlin u.a. (1995), S. 335-359 (343); T. Reinhard, Die geographische Herkunftsangabe nach dem Markengesetz, Würzburg (1998), S. 24

[14] Vgl. $\S 16$ Abs. 2 WbezG (RGBl. (1894), S. 441); $\S 1$ Abs. 3 UWG (1896) (RGBl. (1896), S. 145); 55 Abs. 1 UWG (1909) (RGBl. (1909), S. 499)

[15] Erläuterungen zum Weingesetz vom 7. April 1909, in: Sten. Ber. der Verhandlungen des Deutschen Reichstags, 12. Leg., 1. Session, 248, (1907/1909), Anlage Nr. 987, S. 15; W. Coermann, Weingesetz vom 7. April 1909 nebst den Ergänzungsgesetzen und den Ausführungsbestimmungen des Reichs, Düsseldorf (1909), S. 47; C. Finger, Reichsgesetz gegen den unlauteren Wettbewerb, 4. Aufl., Berlin (1911), S. 96; L. Fuld, Herkunftsbezeichnungen für Weine, in: GRUR (1909), S. 268-274 (268); A. Günther/R. Marschner, Weingesetz vom 7. Juli 1909, Berlin (1910), S. 129. W. Brogsitter, Der Rechtsschutz geographischer Herkunftsbezeichnungen von Weinen im deutschen und französischen Recht, Neustadt (1965), S. $27 \mathrm{f}$

[16] Die Mitglieder des Reichstags. Biographische Notizen, in: Bureau des Reichstags (Hrsg.), ReichstagsHandbuch, 12. Leg. Berlin (1907), S. 204-412 (219, 378)

[17] RGBl. (1909), S. 393

[18] RGBl. (1909), S. 393

[19] Der Begriff "Gemarkung" ist eine Bezeichnung eines Gemeindebezirks, in dem die Rebstöcke des Weins stehen; F. Schoonmaker, Art. "Gemarkung", in: ders., Das Wein-Lexikon, 2. Aufl., Frankfurt a. M. u.a. (1969), S. 109

[20] Der Begriff "Lage" meint bei Weinen einen bestimmten Weinberg oder Weingarten, u.U. mit Parzellen unterschiedlicher Eigentümer. Er entspricht dem burgundischen "Climat" und dem "Cru" in Bordeaux; F. Schoonmaker, Art. "Lage", in: ders., Das Wein-Lexikon, 2. Aufl., Frankfurt a. M. u.a. (1969), S. 139

[21] OLG Darmstadt, abgedruckt in Goldschmid, Weingesetz vom 25. Juli 1930 nebst Ausfüh rungsbestimmungenvom 16. Juli 1932, 2. Aufl., Mainz (1932), S. $218 \mathrm{f}$

[22] RGB1. (1919), S. 687 (1084 f.)

[23] Die französische Gegenseitigkeitserklärung ist abgedruckt in Anlage 2 des Entwurfs eines Gesetzes zur Änderung des Weingesetzes, Sten. Ber. der Verhandlungen des Deutschen Reichstags, 1. Wahlperiode, 376 (1920/24), Anlage Nr. 5458, S. 3 f

[24] Die portugiesische Gegenseitigkeitserklärung ist abgedruckt in Anlage 2 des Entwurfs eines Gesetzes zur Änderung des Weingesetzes, Sten. Ber. der Verhandlungen des Deutschen Reichstags, 1.
Wahlperiode, 376 (1920/24), Anlage Nr. 5458, S. 4

[25] Gesetz zur Unterdrückung des Betruges im Verkehr mit Wein vom 1. August 1905, abgedruckt bei: A.Günther, Die Gesetzgebung des Auslandes über den Verkehr mit Wein, Berlin (1910), S. 52; die dazugehörige Ausführungsverordnung vom 3. Oktober 1907, abgedruckt ebenda, S. 61; und die Verordnung vom 25. Mai 1909, abgedruckt ebenda, S. 71; Verordnung vom 18. September 1909, abgedruckt ebenda, S. 74

[26] Vgl. VO Nr. 218 von 1913 für die Bezeichnung "Madeirawein" und VO 7934 von 1921 für die Bezeichnung "Portwein". Ein Auszug der Verordnungen ist abgedruckt in Anlage 4 des Entwurfs eines Gesetzes zur Änderung des Weingesetzes, Sten. Ber. der Verhandlungen des Deutschen Reichstags, 1. Wahlperiode, 376 (1920/24), Anlage Nr. 5458, S. 4. Dass es sich bei dieser Verordnung um eine portugiesische und nicht um eine deutsche handelt, ergibt sich aus der Begründung zum Entwurf eines Gesetzes zur Änderung des Weingesetzes, ebenda, S. 2

[27] RGB1. I (1923), S. 107

[28] A. Lobe, Die Behandlung des unlauteren Wettbewerbs im Friedensvertrag von Martin Wasserman, in: JW (1920), S. 611 f. (611); ders., Die Bekämpfung des unlauteren Wettbewerbs und die Friedensbedingungen, in: Deutscher Außenhandel, 19. Jg. (1919), Nr. 13/14, S. 160-164 (163); M. Wassermann, Die Behandlung des unlauteren Wettbewerbs im Friedensvertrag, in: MuW 19 (1919/20), S. 99107 (106); ders., Die Behandlung des unlauteren Wettbewerbs im Friedensvertrag, Berlin (1920), S. 117 f.; ders., Deutschland und das Madrider Abkommen betreffend die Unterdrückung falscher Herkunftsbezeichnungen, in: MuW 24 (1924/25), S. 21-28 (27)

[29] RGB1. II (1925), S. 115

[30] W. Borgius, [Über den Schutz geographischer Herkunftsangaben], in: Geographische Herkunftsbezeichnungen im Warenverkehr. Stenographisches Protokoll der vom Handelsvertragsverein am 18. Oktober 1912 zu Berlin veranstalteten Sachverständigen-Konferenz, Berlin (1912), S. 21-31 (27); L. Fuld, Herstellungsort bei Kognak, in: GRUR (1901), S. 94-98 (95); A. Günther/R. Marschner, Weingesetz vom 7. Juli 1909, Berlin (1910), S. 243. Vgl. auch W. Brogsitter Der Rechtsschutz geographischer Herkunftsbezeichnungen von Weinen im deutschen und französischen Recht, Neustadt (1965), S. 54; H. Hieronimi, Weingesetz, 2. Auflage, München u.a. (1958), S. 348; T. Tresper, Die Ursprungsbezeichnung "Champagner" im deutschen Recht, in: MarkenR (2003), S. 349-353 (350)

[31] H. Dormann, Der Schutz geographischer Herkunftsangaben bei Wein in Deutschland, in: ZGE (2014), S. S.468-488 (481 ff.)

[32] Gesetz zur Unterdrückung des Betruges im Verkehr mit Wein vom 1. August 1905, abgedruckt bei: A. Günther, Die Gesetzgebung des Auslandes über 
den Verkehr mit Wein, Berlin (1910), S. 52; die dazugehörige Ausführungsverordnung vom 3. Oktober 1907, abgedruckt ebenda, S. 61; und die Verordnung vom 25. Mai 1909, abgedruckt ebenda, S. 71; Verordnung vom 18. September 1909, abgedruckt ebenda, S. 74

[33] Anders als in der aktuellen Forschungsliteratur teilweise behauptet, stellte die deutsche Kennzeichnungspraxis von deutschem Schaumwein kein Hindernis für den Beitritt zum Madrider Herkunftsabkommen dar. Die Bezeichnung "Champagner" durfte im Deutschen Reich spätestens seit Beginn des 20. Jahrhunderts ausschließlich als Herkunftsangabe für einen aus der région de Champagne stammenden Schaumwein verwendet werden, vgl auch H. Dormann, Der Schutz geographischer Herkunftsangaben bei Wein in Deutschland, in: ZGE (2014), S.468-488 (481 ff.)

[34] Vgl. ausführlich zum zweigleisigen Schutz geographischer Herkunftsangaben, insbesondere im französischen Recht, H. Kickler, Die Geschichte des Schutzes geographischer Herkunftsangaben in Deutschland, Tübingen 2012, S. 359, $401 \mathrm{f}$

[35] F.-K. Beier, Der Schutz geographischer Herkunftsangaben in Deutschland, in: GRUR (1963), S. 169$181 ; 236-245$ (171)

[36] Das LUA ist abgedruckt in: GRUR Ausl. 1959, S. $135 \mathrm{f}$

[37] BGB1. (1961) II, S. 23

[38] BGB1. (1965) II, S. 156

[39] BGBl. (1965) II, S. 176

[40] BGBl. (1969) II, S. 139

[41] BGBl. (1972) II, S. 110

[42] Der deutsch-österreichische Vertrag ist abgedruckt bei K.-H. Fezer, Markengesetz, 3. Aufl. (2001), S. 1735-1741

[43] K.-H. Fezer, Kommentar zum Markengesetz, 4. Aufl. (2009), Vorb $\S 126$, Rn. 44

[44] So der Wortlaut von Art. 4 Abs. 2 des deutschfranzösischen Abkommens zum Schutz geographischer Bezeichnungen, RGB1. II (1961), S. 23

[45] F.-K. Beier, Der Schutz geographischer Herkunftsangaben in Deutschland, in: GRUR Ausl. (1963), S. 169-181; 237-245 (177); ders., Schutz geographischer Herkunftsangaben, in: Das Recht des unlauteren Wettbewerbs in den Mitgliedstaaten der Europäischen Wirtschaftsgemeinschaft, München 3 (1968), S.463-563 (557-563); A. Krieger, Möglichkeiten für eine Verstärkung des Schutzes deutscher Herkunftsangaben, in: GRUR (1960), S. 400-420 (404-419); Der internationale Schutz. von geographischen Bezeichnungen aus deutscher Sicht, in: GRUR Int. (1984), S. 71-89 (75); ders., Zur Auslegung der zweiseitigen Abkommen über den Schutz geographischer Bezeichnungen, in: GRUR Ausl. (1964), S. 499-509; M. Kühn, Zum Schutzumfang geographischer Bezeichnungen nach den zweiseitigen Verträgen über den Schutz von Herkunftsangaben, in: GRUR Int. (1967), S. 268-271

[46] VO (EWG) NR. 816/70, AB1. Nr. L 99, S. 1 vom 5.5.1970, S. 1; VO (EWG) Nr. 2133/74, ABl. Nr. L 227 vom 17.8.1974, S. 1; VO (EWG) Nr. 1475/77, ABl. Nr. L 164 vom 2.7.1977, S. 1; VO (EWG) Nr. 2392/89, AB1. Nr. L 232 vom 9.8.1989, S. 13

[47] ABl. Nr. L 208 vom 24.7.1992, S. 1

[48] ABL. Nr. L 148 vom 06.06.2008, S. 1

[49] ABL. Nr. L 93 vom 31.03.2006, S. 12

[50] ABL. Nr. L 343 vom 14.12.2012, S. 1

[51] ABL. Nr. L 39 vom 13.02.2008, S. 16

[52] ABL. Nr. L 347 vom 20.12.2013, S. 671

[53] So auch: P. Ströbele, F. Hacker, Markengesetz, 10. Auflage (2012), § 12, Rn. 48

[54] EuGH, in: GRUR Int. (1993), S. 76-82 (78), Rn. 28

[55] EuGH, in: GRUR Int. (1999), S. 443-446 (444), Rn. 18

[56] W. Büscher, Der Schutz geographischer Herkunftsangaben nach der Warsteiner-Entscheidung, in: Festschrift für Willi Erdmann zum 65. Geburtstag, Hrsg.: H.-J. Ahrens u.a. Köln u.a. (2002), 237, 239 ff.; R. Knaak, Der Schutz geographischer Angaben nach der EG-Verordnung Nr. 2081/92, in: Festschrift für Gerhard Schricker zum 70. Geburtstag, Hrsg.: A. Ohly u.a., München (2005), S. 815-826, 818

[57] EuGH, in: GRUR (2010), 143-151 (149 f.), Rn. 107129; in: GRUR (2011), 240-242 (242), Rn. 59

[58] P. Ströbele/F. Hacker, Markengesetz, 10. Auflage (2012), § 126, Rn. 47 